\title{
Orbital bi-stripes in highly doped bilayer manganites
}

\author{
T. A. W. Beale, P. D. Spencer, and P. D. Hatton* \\ Department of Physics, University of Durham, Rochester Buildings, South Road, Durham, DH1 3LE, United Kingdom \\ S. B. Wilkins \\ European Commission, Joint Research Center, Institute for Transuranium Elements, Hermann von Helmholtz-Platz 1, \\ 76344 Eggenstein-Leopoldshafen, Germany and European Synchrotron Radiation Facility, Boîte Postal 220, F-38043 Grenoble Cedex, \\ France \\ M. v. Zimmermann \\ Hamburger Synchrotronstrahlungslabor (HASYLAB) at Deutsches Elektronen-Synchrotron (DESY), Notkestraße 85, \\ D-22603 Hamburg, Germany \\ S. D. Brown \\ European Synchrotron Radiation Facility, Boîte Postal 220, F-38043 Grenoble Cedex, France \\ D. Prabhakaran and A. T. Boothroyd \\ Department of Physics, University of Oxford, Clarendon Laboratory, Parks Road, Oxford, OX1 3PU, United Kingdom \\ (Received 2 December 2004; revised manuscript received 6 May 2005; published 16 August 2005)
}

\begin{abstract}
We present high-resolution high-energy and resonant x-ray-diffraction results from $\mathrm{La}_{2-2 x} \mathrm{Sr}_{1+2 x} \mathrm{Mn}_{2} \mathrm{O}_{7}$ for $x=0.55,0.575$, and 0.60 . These compounds show superlattice reflections at wave vectors of $(h \pm \delta, k \pm \delta, l)$ and $(h \pm 2 \delta, k \pm 2 \delta, l)$, arising from orbital ordering with associated Jahn-Teller distortions and charge ordering, respectively. We observe a phase transition boundary between the $x=0.55$ and $x=0.575$ doping levels. Samples with $x=0.55$ display structural characteristics similar to those previously reported for $x=0.5$. Compared to this the long-range order in samples with $x=0.55$ and $x=0.6$ have a distinct change in wave-vector and correlation length. We attribute this to a new orbital bi-stripe phase, accompanied by weak, frustrated, charge ordering. The observed azimuthal dependence of the orbital order reflection supports the model proposed for this new phase.
\end{abstract}

DOI: 10.1103/PhysRevB.72.064432 PACS number(s): 75.47.Lx, 61.10. -i, 61.44.Fw, 71.27.+a

\section{INTRODUCTION}

The $n=2$ member of the Ruddleston-Popper family of manganites forms a bilayer crystal with the general formula $\mathrm{La}_{2-2 x} \mathrm{Sr}_{1+2 x} \mathrm{Mn}_{2} \mathrm{O}_{7}$. The crystal has a layered structure consisting of two $\mathrm{MnO}$ layers separated by a rock-salt-type layer of (La,Sr)O. The result of this layering is an extremely twodimensional crystal with $a=b=3.87 \AA$ and $c=19.95 \AA$ (Fig. 1).

The $\mathrm{La}_{2-2 x} \mathrm{Sr}_{1+2 x} \mathrm{Mn}_{2} \mathrm{O}_{7}$ system presents a very complicated phase diagram. The discovery of colossal magnetoresistance in the $x=0.4$ bilayer $^{1}$ triggered a flurry of interest in this compound. ${ }^{2}$ Following this, numerous studies have been conducted on the $x=0.5$ doping level, showing strong charge and orbital order. Far less work has been done on the overdoped crystals; however, the work that has been done shows many interesting effects. Neutron studies reported that there exists a number of distinct magnetic phases ${ }^{3}$ throughout the stochiometric range (Fig. 2). In the area surrounding the halfdoped region $0.46 \leqslant x \leqslant 0.66$, the low-temperature ordering consists of a type-A antiferromagnetic phase. Above this there appears to be a gap where there exists no long-range magnetic or charge order. This is unique to the bilayer system and is not observed either in the single-layered, or cubic, manganites. Above $x=0.74$ long-range ordering is reformed, but this time in a type-C/C* magnetic phase. Finally, above $x=0.9$ the system enters a type-G phase.
In this paper we will show data from the upper end of the type-A phase with $0.55<x<0.6$. We have applied resonant and high-energy $\mathrm{x}$-ray diffraction to look at the superlattice peaks associated with the charge and orbital ordering as well as the structural distortions in the compounds with $x=0.55$, 0.575 and $x=0.6$ doping levels. We observe a distinct change between the $x=0.55$ and $x=0.575$ doping levels, characterized by an increase in the phase transition temperature associated with both charge-ordering $(\mathrm{CO})$ and concomitant Jahn-Teller (JT) structural distortions $\left(\mathrm{T}_{C O / J T}\right)$ coupled with a discontinuous change in the ordering wave vector. We also report a strong correlation between the Jahn-Teller distortion peak intensity and the commensurability of the superlattice reflections in the $x=0.575$ and 0.6 samples. The wave vector of the orbital order at low temperature suggests a periodicity five times larger than that of the chemical unit cell in the $a b$ plane. We propose a quasi-bi-stripe phase of orbital order, complemented by weak, frustrated, charge ordering. A charge and orbital order pattern has been constructed and is proposed as the structure in the range $0.55<x<0.625$. Such a structure would have a particular polarization and azimuthal dependence of the orbital order reflection that has been simulated. Our experimental results at the Mn $K$ edge confirm the predicted azimuthal dependence, giving us confidence in the proposed structure observed using both resonant $\mathrm{x}$-ray and high-energy $\mathrm{x}$-ray diffraction. The orbital or- 


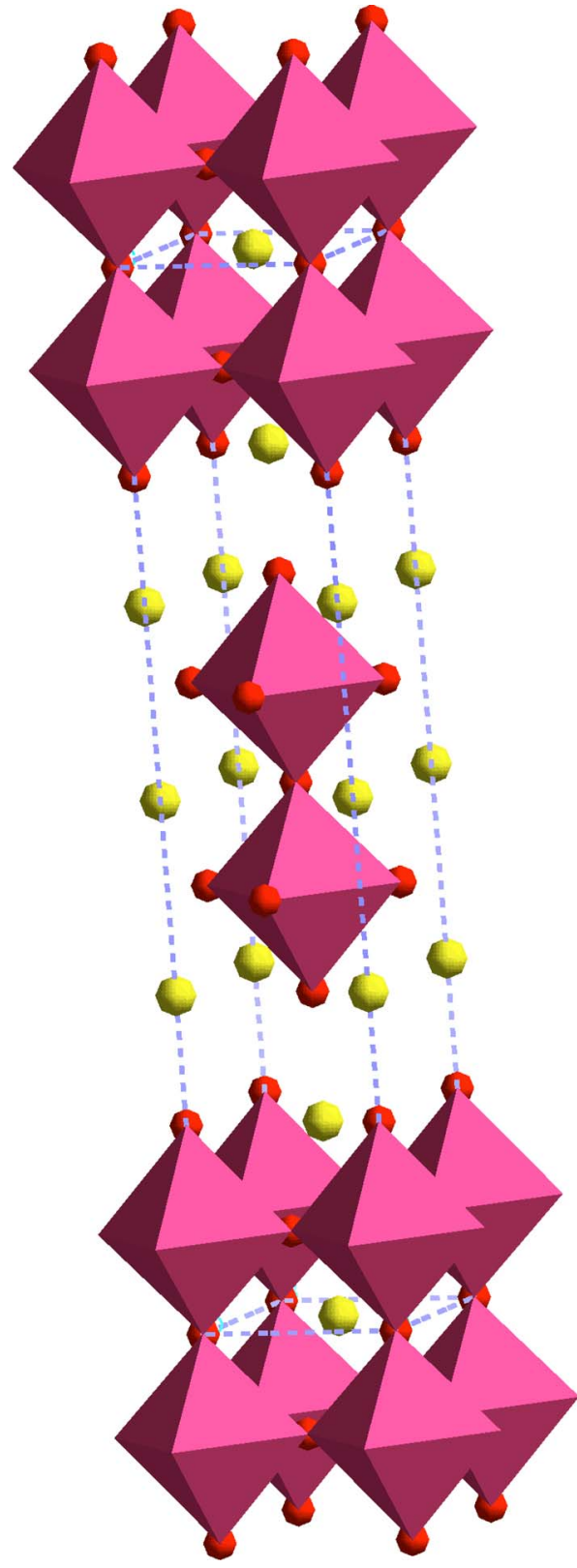

FIG. 1. (Color online) Crystal structure of $\mathrm{La}_{2-2 x} \mathrm{Sr}_{1+2 x} \mathrm{Mn}_{2} \mathrm{O}_{7}$. Purple octahedra respresent $\mathrm{MnO}_{6}$ octahedra, yellow spheres represent $\mathrm{La} / \mathrm{Sr}$ sites, and red spheres represent oxygen.

der is accompanied with a structural Jahn-Teller distortion, which appears to be more persistent and stable than in the half-doped bilayer manganites.

\section{EXPERIMENT AND RESULTS}

High-quality single crystals of $\mathrm{La}_{2-2 x} \mathrm{Sr}_{1+2 x} \mathrm{Mn}_{2} \mathrm{O}_{7}$ were grown using the floating zone method at the University of Oxford. ${ }^{5}$ These crystals were prealigned using an in-house $\mathrm{Cu}$ rotating anode system. ${ }^{6}$ Experiments were performed using high-energy $(100 \mathrm{keV}) \mathrm{x}$-ray diffraction and also resonant $\mathrm{x}$-ray scattering at the $\mathrm{Mn} K$ edge.

High-energy x-ray diffraction was performed at the BW5 beamline at HASYLAB, Hamburg. The beamline is

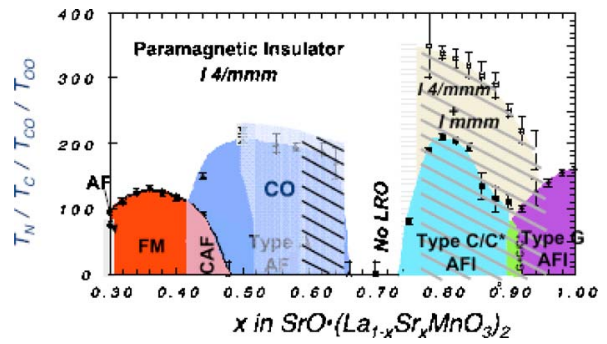

FIG. 2. (Color online) Structural phase diagram from Qui et al. ${ }^{4}$ of the bilayer manganite. The region of interest in this paper is the area with diagonal black stripes around $x=0.6$. We propose a different orbital and charge order to that of the half-doped bilayer.

equipped with a wiggler and a water-cooled $\mathrm{Cu}$ filter to produce $\mathrm{x}$-rays in the spectral range $60-150 \mathrm{keV}$. The analyzer and monochromator were matched SiGe-graded crystals to provide a resolution matched to the sample rocking curve width. The x-ray beam had an incident beam size of $1 \times 1 \mathrm{~mm}$ and an energy of $100 \mathrm{keV}$. Detection was provided by a solid-state detector, gated to remove harmonics using a single-channel analyzer.

The samples were mounted on a the cold finger on an avalanche photodiode (APD) displex cryofurnace capable of a temperature range $10 \mathrm{~K}<T<400 \mathrm{~K}$. The orientation of the sample was such that the $c$ axis was parallel to the incident beam and the $a b$ plane, perpendicular.

Resonant $\mathrm{x}$-ray diffraction was undertaken at the XMaS UK CRG beamline at the ESRF. ${ }^{7}$ An incident beam energy in the region of $6.555 \mathrm{keV}$ ( $\mathrm{Mn} K$ edge) was provided by a double-bounce $\mathrm{Si}(111)$ water-cooled monochromator, with harmonic rejection mirrors. Crystals pre-cleaved with the $c$-axis surface normal were mounted with the $c$-axis along the scattering vector, allowing access to (00l)-type reflections. The sample environment was similar to that at BW5 with a closed-circle cryostat held in a Eulerian cradle. $\mathrm{A} \mathrm{Cu}$ (220) crystal was used for polarization analysis, which at $6.555 \mathrm{keV}$ has a scattering vector $47^{\circ}$ from the incident beam. This allows a leak through of $\sim 3.5 \%$ between the two polarization channels.

We describe our results in the following three sections organized by doping stoichiometry, starting by the composition most similar to the well-characterised $x=0.5$ composition.

$$
\text { A. } x=0.55
$$

The sample was mounted on the high-energy beamline. Upon cooling, the sample below the charge-ordering temperature $\left(\mathrm{T}_{C O}\right)$ superlattice peaks appeared at wave vectors $(h \pm \delta, k \pm \delta, l)$. These superlattice peaks, as have previously been described, ${ }^{8-11}$ arise from Jahn-Teller (JT) structural distortions. They were found regularly throughout reciprocal space with intensities $\sim 15000$ counts per second. Secondary weaker superlattice peaks at $(h \pm 2 \delta, k \pm 2 \delta, l)$, corresponding to charge ordering $(\mathrm{CO})$ of the nominal $\mathrm{Mn}^{3+}$ and $\mathrm{Mn}^{4+}$, were also found. The observed wave vectors of both these peaks require $\delta=0.25$. 
The use of the nomenclature of the position of the peaks, should not be interpreted as the charge-order peak existing as a harmonic of the Jahn-Teller peak. These peaks arise from distinctly different phenomena. The Jahn-Teller satellites at $(\delta, \delta, 0)$-type positions are due to a crystallographic distortion. This scattering is due to a movement of a Mn ion and associated $\mathrm{O}$ atoms. This gives a strong $\mathrm{x}$-ray-scattering signal. The charge-order peak appears at $(2 \delta, 2 \delta, 0)$-type positions. This occurs because of weak modulations in the electron density. Deviations from the average $3.5+$ charge on the manganese ion forms an alternating pattern of $\mathrm{Mn}^{3.5-\delta}$ and $\mathrm{Mn}^{3.5+\delta}$ ions. The former are Jahn-Teller distorted. As such the periodicity of the cooperative Jahn-Teller distortion is locked into a value twice that of the periodicity of the charge order. Because in the highly doped bilayers the wave vector of the satellite peaks varies with temperature we have symbolized their position.

It has been observed by Wilkins et al. ${ }^{9}$ that the chargeorder and orbital-order signals in $\mathrm{La}_{1.05} \mathrm{Sr}_{1.95} \mathrm{Mn}_{2} \mathrm{O}_{7}$ scatter in different polarization channels. The charge-order scattering originates entirely from the $f^{\prime}$ term from the dipole transition. As such, the polarization vector is unrotated throughout the scattering process and exists only in the $\sigma \rightarrow \sigma$ channel. In contrast, the scattering at the orbital-order wave vector originates from the $f^{\prime \prime}$ term. This has a complex polarization rotation and exists in both the $\sigma \rightarrow \sigma$ and $\sigma \rightarrow \pi$ channels. Such observations clearly demonstrate that these scattering signals originate from fundamentally different processes. The structural models proposed to date involving charge- and orbital-ordered manganese ions would produce higher-order harmonics at odd positions (i.e., $1 \tau, 3 \tau$, etc.) if the electrondensity difference were atomistically abrupt. There have been no reports that this is the case; instead, it is believed that there exists a more gradual electron-density profile.

The charge-order satellite peaks were $\sim 10$ times weaker than those of the JT distortions. The peak shapes from both the JT and CO signals displayed a Gaussian line shape (Fig. 3). This suggests that the resolution was instrument limited. Indeed a measurement of the $(2,0,0)$ Bragg peak shows a similar width and shape. The slight difference in the peak width is due to different instrument resolutions in different geometries and is not indicative of intrinsic differences in the correlation lengths. The instrument resolution was purposely set fairly low to maximize the intensity; however, it was sufficient to accurately measure the widths of the superlattice peaks in the higher-doped samples.

The superlattice peaks had a far greater width in the [001] direction. This we attribute to the two-dimensional nature of the crystal structure.

The $x=0.55$ sample was cooled to the base temperature of $12 \mathrm{~K}$, and the JT and $\mathrm{CO}$ peaks were measured upon warming. At each temperature, thermal equilibrium was achieved before the intensity and width were measured. In order to accurately measure the commensurate wave vector, the position of two satellite peaks opposite each other with respect to a Bragg peak was determined. No significant change of the position or peak width was detected throughout the temperature range (Fig. 4). The measured integrated intensity displayed a significant increase at $\sim 120 \mathrm{~K}$ and then reached a maximum at $\mathrm{T}_{N}(180 \mathrm{~K})$. The intensity of the peaks then fell
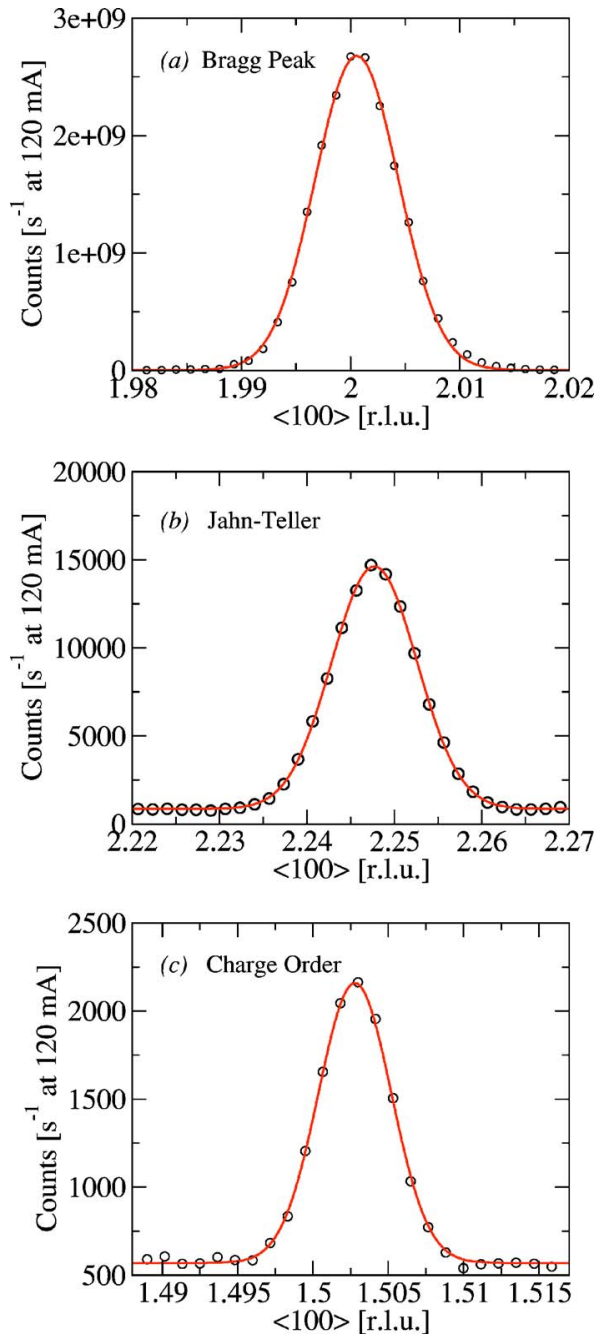

FIG. 3. (Color online) $(2+\delta, \delta, 0)$ The line shapes of the (a) $(2,0,0)$ Bragg peak, (b) the Jahn-Teller $(2-\delta,-\delta, 0)$, and (c) the charge order $(2-2 \delta,-2 \delta, 0)$ peaks taken at $170 \mathrm{~K}$ measured from the $x=0.55$ sample in the [100] direction. The solid lines show Gaussian fits to the data. Errors are within the size of the symbols.

sharply with increasing temperature, until reaching background at $220 \mathrm{~K}$. This behavior is extremely similar to the $x=0.475$ and 0.5 compounds. ${ }^{9}$ We did observe a slight increase in the transition temperatures $\left(\mathrm{T}_{n}, \mathrm{~T}_{C O}\right)$ of about $10 \mathrm{~K}$ compared to that in the $x=0.5$ sample.

\section{B. $x=0.575$}

The only satellite peaks detected by high-energy x-ray diffraction were located at $(h \pm \delta, k \pm \delta, l)$ positions. These peaks associated with JT distortions were significantly weaker than those found in the $x=0.55$ sample. Comparing the relative intensities of the peak strength at $\pm 2 \delta$ with that at $\pm \delta$ in the $x=0.55$ system, a similarly proportioned signal in the $x=0.575$ sample would have been extremely difficult to detect. As such we suspect that charge ordering does exist, but it is too weak for us to detect. The peak at $(2-\delta,-\delta, 0)$ was much broader in the $x=0.575$ than in the $x=0.55$ sample, and therefore, the measurements were not limited by the in- 




FIG. 4. (Color online) (a) Measurement of the wave vector of the Jahn-Teller distortion peak at $(h \pm \delta, k \pm \delta, l)$ for the doping levels $x=0.55$ (black triangles), $x=0.575$ (blue circles), and $x=0.6$ (red squares) as a function of temperature upon warming from base temperature. (b,c,d) (middle panel) Integrated intensity of the JahnTeller peak for the $x=0.55,0.575$, and 0.6 samples. (c) Intensity of the charge order in the $x=0.55$ (black triangles) and $x=0.6$ (red squares) doping levels.

strument resolution. The shape of the peak was Lorentzian squared in the high-resolution [001] direction. This suggests that the instrument resolution makes a negligible contribution to the width of the peak. We observed no significant variation of the peak width with temperature. Unlike the $x=0.55$ sample the Jahn-Teller signal in the $x=0.575$ sample does display a significant variation in the wave vector, $\delta$ [Fig. 4(a)]. This variation follows a strikingly similar pattern to the variation of the intensity of the JT distortion as a function of temperature. Initially at low temperature $\delta \approx 0.2$; however, upon warming and with increasing intensity this value reaches $\delta=0.22$.


FIG. 5. (Color online) Scans of the (a) $(2+\delta, 0-\delta, 0)$ JahnTeller superlattice peak and (b) the $(2-2 \delta,-2 \delta, 0)$ charge-order superlattice peak, in the $x=0.6$ sample at $210 \mathrm{~K}$ measured in the [h00] direction. Solid lines are fits to the data using Lorentzian squared line shapes and a linear background.

\section{C. $x=0.60$}

Satellite peaks were found using high-energy x-ray diffraction at both $(h \pm \delta, k \pm \delta, l)$ and $(h \pm 2 \delta, k \pm 2 \delta, l)$ in the $x=0.60$ sample. The Jahn-Teller peak is significantly stronger than that occurring in either the $x=0.55$ or $x=0.575$ doped samples. The charge-order peak, however, is some 40 times lower in intensity than the Jahn-Teller peak, compared to only 10 times lower in the $x=0.55$ sample. As with the $x=0.575$ sample the peaks are not resolution limited and can be accurately fitted with a Lorentzian-squared line shape (Fig. 5).

The wave vector of the superlattice peak due to the JahnTeller distortion varies considerably [Fig. 4(a)]. As was seen in the sample with $x=0.575$, this follows the general trend of the intensity of the peak [Fig. 4(d)]. In addition the charge order satellite peak shows a similar pattern in the intensity with respect to temperature as the Jahn-Teller peak [Fig. 4(a)].

Resonant diffraction at the Mn $K$ edge of the $x=0.6$ bilayer sample was performed specifically to look at the anisotropy of the structural distortion and concomitant orbital order. There has been much discussion about what $K$ edge diffraction is sensitive to and whether or not it is a direct observation of orbital ordering. There appears to be three possibilities. Either the resonant signal is sensitive to the weak quadropole transition from the $1 s-3 d$, or to the dipole transition into the $4 p$ state, which is influenced by the $3 d$ level through Coulomb repulsion, or finally, that it is sensitive to the dipole transition to the $4 p$, which is effected by the nearest-neighbor bond length and orbital occupation. The 


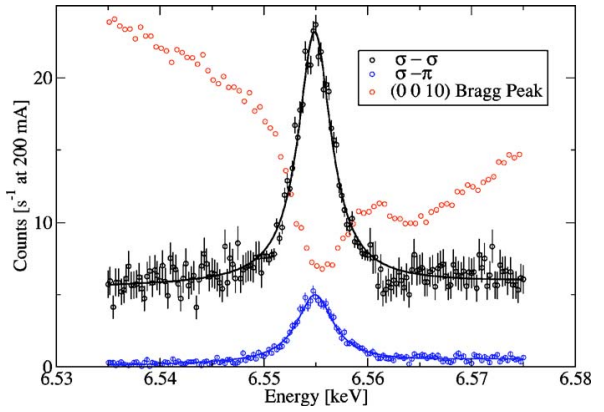

FIG. 6. (Color online) The energy dependence of the orbital signal at the Mn $K$ edge measured in the $\sigma-\sigma$ and $\sigma-\pi$ channels. Solid lines show a Lorentzian fit. The scaled energy dependence of the $(0,0,10)$ Bragg peak for comparison with the Mn $K$ absorption edge.

first possibility seems unlikely as one would expect a difference in energy between the quadropole and dipole magnetic signals, and as in $\mathrm{V}_{2} \mathrm{O}_{3},{ }^{12}$ it would be expected that both would be visible. Elfimov et al. ${ }^{13}$ have suggested that the Coulomb interaction proposed by Ishihara et al. ${ }^{14}$ is unlikely, and the consensus seems to be that the final option is most probable. As such, we are not looking at a direct probe of the orbital order, we are looking at the cooperative Jahn-Teller distortion, which accompanies the orbital ordering. The separation of these two phenomena does appear to be possible using $L$-edge diffraction where it has been demonstrated in $\mathrm{La}_{0.5} \mathrm{Sr}_{1.5} \mathrm{MnO}_{4} \cdot{ }^{15,16}$

The resonant signal of the $(\delta, \delta, 10)$ was collected, which was found to resonate in both the $\sigma-\sigma$ and $\sigma-\pi$ channels (Fig. 6). These resonances occurred at the same energy as the absorption edge measured at the $(0,0,10)$ Bragg peak and have Lorentzian line shapes.

The cross section for resonant scattering from an electrondipole transition $(E 1)$ can be written as follows:

$$
f_{E 1}^{\mathrm{xres}}=f_{0}+i f_{1}+f_{2} \text {, }
$$

where the terms $f_{n}$ are given by

$$
\begin{gathered}
f_{0}=\left(\hat{\boldsymbol{\epsilon}}^{\prime} \cdot \hat{\boldsymbol{\epsilon}}\right)\left[F_{11}+F_{1-1}\right], \\
f_{1}=-\left(\hat{\boldsymbol{\epsilon}}^{\prime} \cdot \hat{\boldsymbol{\epsilon}}\right) \cdot \hat{z}\left[F_{11}-F_{1-1}\right], \\
f_{2}=\left(\hat{\boldsymbol{\epsilon}}^{\prime} \cdot \tilde{T} \cdot \hat{\boldsymbol{\epsilon}}\right)\left[2 F_{10}-F_{11}-F_{1-1}\right],
\end{gathered}
$$

where $\hat{\boldsymbol{\epsilon}}$ and $\hat{\boldsymbol{\epsilon}}^{\prime}$ are the polarization vectors of the incident and scattered beam, respectively, $\hat{z}$ is a unit vector in the direction of the magnetic moment, and $\widetilde{T}$ is the scattering tensor.

For $\sigma$ polarized incident light, one would only expect a signal to be present in the $\sigma-\pi$ channel, if the signal originates from the $f_{1}$ term. However, the presence of $\sigma-\sigma$ scattering indicates that the resonance occurs because of terms in $f_{0}$ or $f_{2}$. As terms in $f_{0}$ are independent of $\vec{Q}$, we believe the resonance to occur solely from the $f_{2}$ term. As expected, these resonances can be fitted satisfactorily with a Lorentzian line shape, typical of a dipole transition. The center of these resonances occurs at $6.555 \mathrm{keV}$, corresponding to the $\mathrm{Mn}$ absorption edge observed of the $(0,0,10)$ Bragg peak. This resonant energy is identical to that seen in $\mathrm{La}_{1-x} \mathrm{Ca}_{x} \mathrm{MnO}_{3}$ by XANES by Bridges et al., ${ }^{17}$ who attributed this main peak to a dipole transition. They also observed weak pre-edge features $\sim 15 \mathrm{eV}$ below this, which could either be due to forbidden quadropole transition or hybridization of the $4 p$ level. We did not observe these peaks by diffraction in the bilayer; however, they could be within our noise level.

The azimuthal angle dependence was collected by measuring the integrated intensity of the superlattice peak in each polarization channel for a given azimuthal angle. Because of the simultaneous presence of a signal in both polarization channels we have calculated the polarization of the scattered $\mathrm{x}$-ray beam by the Stokes parameter as defined by

$$
P_{1}(\text { Stokes parameter })=\frac{I_{\sigma-\sigma}-I_{\sigma-\pi}}{I_{\sigma-\sigma}+I_{\sigma-\pi}} .
$$

This has the effect of self-normalization and removes any effect of angular changes in the size of the geometric beam footprint. The integrated intensity of the signal in either channel was measured through a scan of the polarization analyzer angle $\theta$. It should be noted that the Jahn-Teller structural distortion and the orbital ordering have the same symmetry around the $\mathrm{Mn}^{3+}$ ion. As such this azimuthal dependence is valid for both phenomena, independant of any sensitivity arguments.

A model of the charge and orbital order has been constructed for the highly doped phase (Fig. 7). This model was constructed in such a way to agree with the fivefold periodicity. Although our model implies an integral step in the electron density, corresponding to a square wave-type model. In reality the electron density would be smooth, providing a sinusoidal charge density. This would, therefore, not display any higher-order harmonics. Using this model the azimuthal dependence has been calculated by using the anisotropy of the tensor of susceptibility (ATS) method. On resonance, the scattering on a manganese site is given by a tensor $\widetilde{T}$ due to the local site symmetry $D_{4 h} \cdot{ }^{18}$ We therefore calculated the total structure factor from all manganese sites for the reflection $(0.2,0.2,10)$ in the unit to obtain a single scattering tensor $\widetilde{T}_{00}$. The structure factor can then be calculated by

$$
\widetilde{T}_{00}=\sum_{i=1}^{4} \widetilde{T}_{i}^{D_{4 h}} e^{i \vec{Q} \cdot \vec{r}_{i}},
$$

where $\vec{Q}$ is the scattering vector and $\vec{r}_{i}$ are the positions of each of the four $\mathrm{Mn}^{3+}$ ions.

The intensity can therefore be calculated using the following formula:

$$
I=\left|\hat{\epsilon}^{\prime} \cdot \widetilde{T}_{00} \cdot \hat{\epsilon}\right|^{2} .
$$

The azimuthal dependence of the $(\delta, \delta, 10)$ superlattice peak is displayed in (Fig. 8), together with the results of the simulation. The experimentally determined Stokes parameter does not fall to -1 as predicted by the ATS simulation. This is because of the $\sigma-\sigma$ signal being much larger than the $\sigma-\pi$, and therefore, even a relatively small $\sigma-\sigma$ signal dramatically increases the parameter value. This small $\sigma-\sigma$ sig- 


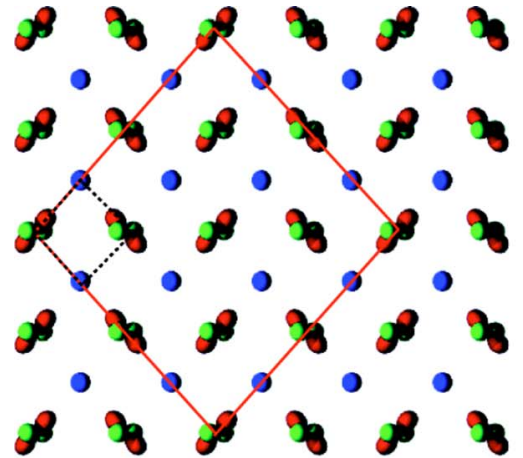

a)



b)

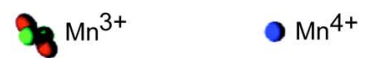

FIG. 7. (Color online) (a) The accepted charge and orbital ordering of $\mathrm{La}_{2-2 x} \mathrm{Sr}_{1+2 x} \mathrm{Mn}_{2} \mathrm{O}_{7}$ with $x=0.5$ (b) Proposed charge and orbital ordering for the $x=0.6$ doping level. In both diagrams only the manganese ions are shown for clarity. The chemical unit cell is shown by the black dotted line, and the orbital order super-cell shown by the solid red line. The orbitals are displayed as $d_{x^{2}+y^{2}}$ as these have been shown to be dominant by ab-initio calculations ${ }^{2}$.

nal could be due to a small amount of background scatter present in this channel. Overall though, there is a general agreement between the fit and the data, suggesting that a correct model of the orbital anisotropy has been used.

The measurements of the azimuthal dependence of the orbital order is a method of testing the order model. Different orbital patterns result in a different azimuthal dependence and the strong agreement between calculated and measured values corresponds to this model. A similar study has been reported by Di Matteo et al. ${ }^{10}$ on the $x=0.5$ doped bilayer. In their paper they simulated the traditional Jahn-Teller distorted checkerboard pattern and found an excellent agreement with their experimental data.

\section{DISCUSSION}

The distinct change in the behavoir of the bilayer suggests that the higher doping, where $x \geqslant 0.575$ (highly doped re-



FIG. 8. (Color online) The azimuthal dependence of the Stokes parameter as calculated using the structure proposed in Fig 7 and equations 5 and 6 (solid line) and the experimentally determined intensity of orbital order signal measured in the $\sigma-\sigma$ and $\sigma-\pi$ channels.

gion) forms a second subphase within the charge-ordered regime. Here the JT distortion, and most probably the charge order, display strikingly different behavior than that observed for $x \leqslant 0.55$ (mid-doped region). The behavior of the $x=0.55$ sample can be seen to be very similar to the lower dopings by comparing with the results presented by Wilkins et al. ${ }^{9}$ The interaction between the AFM order below $\mathrm{T}_{N}$ and the orbital and charge ordering appears to be unique to the bilayer manganites. The onset of the AFM ordering simultaneously reduces the intensity of the in-plane ordering. It was originally thought that the $\mathrm{CO}$ completely collapsed ${ }^{19}$ and then was a reentrant again at lower temperatures. This collapse appears to be incomplete and any increase at low temperatures is very small. Similar behavoir was also seen in the $x=0.475$ and 0.5 samples. ${ }^{9}$ It has been suggested that a spin freezing occurs below $100 \mathrm{~K},{ }^{20}$ which corresponds to the minimum in the charge-order reflection intensity. This suggests that there are spin fluctuations occuring below $\mathrm{T}_{N}$ that gradually slow to form a spin-frozen state at $100 \mathrm{~K}$.

Unlike previous reports, ${ }^{11,21}$ we do not observe a smooth transition of the wave vector with doping level. It was suggested that $\delta$ follows the trend $\delta=(1-x) / 2$; however, we observe discrete changes in the wave vector, and, in particular, the wave vector is not stable throughout the temperature range of the charge-ordered regime. Although this trend undoubtedly seems true, in general, we suggest that $\delta$ moves to the closest stable commensurate position (see Table I).

The enhanced stability of the JT distorted phase was shown before by Campbell et al. ${ }^{22}$ however, no explanation was given for this. We present a model showing a stable ordering of the JT distortions around the $x=0.6$ doping; however, this does not explain why it is stable to a higher temperature than the $x=0.5$ structure. Indeed, it is interesting that the distortions in the high-doping region appear to have a much shorter correlation length (see Table I and Fig. 9) than those in the mid-doped region, and yet the superlattice peaks persevere to a higher temperature. The significant change in correlation length between the $x=0.55$ and the $x=0.575$ adds evidence for a phase transition between these doping levels.

The mid-doped $0.475<x<0.55$ region showed very little change in the wave vector of the superlattice peaks, the po- 
TABLE I. Position and inverse correlation lengths of the JahnTeller peaks with respect to the doping of the sample. Inverse correlation lengths are measured at peak intensity, $\delta$ is taken at base temperature.

\begin{tabular}{ccc}
\hline \hline$x$ & $\begin{array}{c}\text { Position at } 10 \mathrm{~K} \\
(\delta)\end{array}$ & $\begin{array}{c}\text { Inverse correlation length } \\
\left(10^{-2} \AA^{-1}\right)\end{array}$ \\
\hline $0.475^{\mathrm{a}}$ & 0.25 & 0.19 \\
$0.5^{\mathrm{a}}$ & 0.25 & 0.19 \\
0.55 & 0.25 & $\leqslant 0.6$ \\
0.575 & 0.20 & 2.3 \\
0.6 & 0.20 & 1.4 \\
\hline \hline
\end{tabular}

Data taken from Reference 9.

sition of which is adequately explained using the checkerboard and CE-type model. In the higher-doped structure, this symmetry breaks down and the positions of the superlattice peaks align at incommensurate values. In addition, these values change as the intensity changes with temperature. This direct relation between the propagation vector of the superlattice cell and the amplitude of that cell has not been seen to this extent in other manganite. It suggests a dynamic stripe system that changes configuration as the degree of charge segregation alters. In order to understand this ordered system, we first have to understand the origin of the lowtemperature propagation wave vector.

The highly doped charge-order phase seems to be less correlated than that displayed by the mid-doped samples. The inverse correlation length of both the JT and charge ordering (calculated through the width of the superlattice peaks) remain constant at all temperatures, for each of the samples. The JT superlattice peak in the $x=0.55$ sample had a maximum inverse correlation length of $\zeta \leqslant 6.3 \times 10^{-3} \AA^{-1}$, whereas the JT peaks from $x=0.575$ and $x=0.6$ doped samples were $\zeta=2.3 \times 10^{-2} \AA^{-1}$ and $\zeta=1.4 \times 10^{-2} \AA^{-1}$, respectively. This difference is clearly shown in Fig. 9, where the $x=0.55$ sample JT peak is fitted to a Gaussian line shape, and the much broader JT peaks from the highly doped phase are fitted to Lorentzian squared line shapes.

Substantial discussion was generated after the original discovery of the charge-ordered systems in the $A B \mathrm{MnO}_{3}$

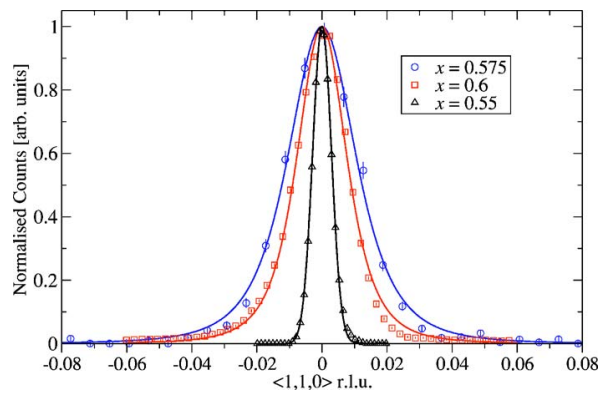

FIG. 9. (Color online) Comparison of the Jahn-Teller superlattice peaks in the $x=0.55,0.575,0.60$ samples. The solid line shows a Lorentzian squared fit for the $x=0.575$ and 0.60 samples, and a Gaussian fit for the $x=0.55$ sample (see section II A). The intensities of the peaks have been normalised, and the centre of the fits set to zero. All the linear backgrounds have been removed after fitting the data. compounds, as to the relative merits of the bi-stripe ${ }^{23}$ (BS) and Wigner ${ }^{24}$ crystal (WC) models. ${ }^{25-27}$ These models provided solutions to the orbital ordering of compounds where the ratio of nominally $\mathrm{Mn}^{3+}$ and $\mathrm{Mn}^{4+}$ is not $1: 1$. These same arguments can be applied to the bilayer crystal. Here it appears that we have an ordering that lies midway between these models and the checkerboard pattern. The lowest stable BS and WC models have $x=0.66$, with an orbital orbit propagation vector $(2 \pi / a)\left(\frac{1}{3}, 0,0\right)$. Here we have a doping level less than this, and the position of the superlattice peaks displays a larger propagation vector. A satisfactory model of this midpoint is displayed in Fig. 7. This 2-1 stripe model has similarities to both models mentioned above. The slipage distance between successive $\mathrm{Mn}^{3+}$ ions alters, as one can see in the BS model, however the correlation in these species in the [110] direction, is similar to the WC system. The opposition of the direction of the orbitals of the stripe pairs is necessary for the observed wave vector from the JT superlattice. This can be understood as a simple exchange of the orbitals between a single $\mathrm{Mn}^{4+}$ and superexchange between two nominal $\mathrm{Mn}^{4+}$ ions.

Recently, Maitra and Taraphder ${ }^{28}$ have considered the magnetic and orbital ordering in the overdoped bilayer manganites; however, they did not consider a bi-stripe model. Very recently an electron diffraction study of the higher doped bilayer manganites has been conducted by Luo et al. ${ }^{29}$ This reaches the same conclusion as our study regarding the charge and orbital pattern that exists in these samples.

Increasing the temperature of the sample alters both the intensity and the incommensurate state of the charge-ordered peaks of the highly doped phase. The wave vector $\delta$ increases, however does not reach the stable mid-doping value of $\delta=0.25$. As $\delta$ increases we can imagine the 2-1 stripe model gradually turning into the mid-doped stripe phase by losing double stripes. The incommensurability reaches a point of maximum intensity where every other double stripe is now a single stripe, and therefore, instead of a double stripe and then a single stripe repeated, there is now a double stripe and then two single stripes before a second double stripe. It would be expected that this model would not be well correlated as there are not sufficient $\mathrm{Mn}^{3+}$ ions. As the temperature increases further and the intensity decreases, $\delta$ falls back to the $\delta-0.2$ highly doped phase value.

\section{CONCLUSIONS}

We have presented results from high-resolution $\mathrm{x}$-rayscattering studies of $\mathrm{La}_{1+2 x} \mathrm{Sr}_{2-2 x} \mathrm{Mn}_{2} \mathrm{O}_{7}$ where $x=0.55,575$, and 0.6. It is clearly demonstrated that there is a distinct change in the nature of the charge ordering and accompanying Jahn-Teller distortion at $0.55<x<0.575$. The $x=0.55$ sample shows very similar behavior to that seen in $x=0.5$, whereas the $x \geqslant 0.575$ samples show ordering with a much lower correlation. A striking incommensurate behaviour is also seen in this more highly-doped charge-ordered phase. A model containing quasi-bi-stripe ordering is proposed, and the measured azimuthal dependence of the orbital order agrees with this model. We suggest that this quasi-bi-stripe ordering would turn into a true bi-stripe order as the doping level is increased further toward $x=0.66$. 


\section{ACKNOWLEDGMENTS}

T.A.W.B. and P.D.S. wish to thank EPSRC for support. P.D.H. thanks the University of Durham Research Founda- tion for support. S.B.W. would like to thank the European Commission for the support in the frame of the "Training and Mobility of Researchers" program.
*Electronic address: p.d.hatton@dur.ac.uk

${ }^{1}$ Y. Moritomo, A. Asamitsu, H. Kuwaha, and Y. Tokura, Nature (London) 380, 141 (1996).

${ }^{2}$ A. Koizumi, T. Nagao, Y. Kakutani, N. Sakai, K. Hirota, and Y. Murakami, Phys. Rev. B 69, 060401 (2004).

${ }^{3}$ C. D. Ling, J. E. Millburn, J. F. Mitchell, D. N. Argyriou, J. Linton, and H. N. Bordallo, Phys. Rev. B 62, 15096 (2000).

${ }^{4}$ X. Qui, S. Billinge, C. Kmety, and J. Mitchell, J. Phys. Chem. Solids 65, 1423 (2004).

${ }^{5}$ D. Prabhakaran and A. T. Boothroyd, J. Mater. Sci.: Mater. Electron. 14, 587 (2003).

${ }^{6}$ S. B. Wilkins, P. D. Spencer, P. D. Hatton, B. K. Tanner, T. A. Lafford, J. Spence, and N. Loxely, Rev. Sci. Instrum. 73, 2666 (2002).

${ }^{7}$ S. D. Brown, L. Bouchenoire, D. Bowyer, J. Kervin, D. Laundy, M. G. Longfield, D. Mannix, D. F. Paul, A. Stunault, P. Thompson et al., J. Synchrotron Radiat. 8, 1172 (2001).

${ }^{8}$ Y. Wakabayashi, Y. Murakami, I. Kayama, T. Kimura, Y. Tokura, Y. Moritomo, K. Hirota, and Y. Endoh, J. Phys. Soc. Jpn. 69, 2731 (2000).

${ }^{9}$ S. B. Wilkins, P. D. Spencer, T. A. W. Beale, P. D. Hatton, M. von Zimmermann, S. D. Brown, D. Prabhakaran, and A. T. Boothroyd, Phys. Rev. B 67, 205110 (2003).

${ }^{10}$ S. D. Matteo, T. Chatterji, Y. Joly, A. Stunault, J. A. Paixao, R. Suryanarayanan, G. Dhalenne, and A. Revcolevschi, Phys. Rev. B 68, 024414 (2003).

${ }^{11}$ J. Q. Li, C. Dong, L. H. Liu, and Y. M. Ni, Phys. Rev. B 64, 174413 (2001).

${ }^{12}$ L. Paolasini, C. Vettier, F. de Bergevin, F. Yakhou, D. Mannix, A. Stunault, W. Neubeck, M. Altarelli, M. Fabrizio, P. A. Metcalf, et al., Phys. Rev. Lett. 82, 4719 (1999).
${ }^{13}$ I. S. Elfimov, V. I. Anisimov, and G. A. Sawatzky, Phys. Rev. Lett. 82, 4264 (1999).

${ }^{14}$ S. Ishihara and S. Maekawa, Phys. Rev. Lett. 80, 3799 (1998).

${ }^{15}$ C. W. M. Castleton and M. Altarelli, Phys. Rev. B 62, 1033 (2000).

${ }^{16}$ S. B. Wilkins, P. D. Spencer, P. D. Hatton, S. P. Collins, M. D. Roper, D. Prabhakaran, and A. T. Boothroyd, Phys. Rev. Lett. 91, 167205 (2003).

${ }^{17}$ F. Bridges, C. H. Booth, G. H. Kwei, J. J. Neumeier, and G. A. Sawatzky, Phys. Rev. B 61, R9237 (2000).

${ }^{18}$ P. Carra and B. T. Thole, Rev. Mod. Phys. 66, 1509 (1994).

${ }^{19}$ J. Dho, W. S. Kim, H. S. Choi, E. O. Chi, and N. H. Hur, J. Phys.: Condens. Matter 13, 3655 (2001).

${ }^{20}$ A. I. Coldea, S. J. Blundell, C. A. Steer, J. F. Mitchell, and F. L. Pratt, Phys. Rev. Lett. 89, 277601 (2002).

${ }^{21}$ S. Larochelle, A. Mehta, N. Kaneko, P. K. Mang, A. F. Panchula, L. Zhou, J. Arthur, and M. Greven, Phys. Rev. Lett. 87, 095502 (2001).

${ }^{22}$ B. J. Campbell, D. N. Argyriou, J. F. Mitchell, R. Osborn, B. Ouladdiaf, and C. D. Ling, Phys. Rev. B 69, 104403 (2004).

${ }^{23}$ S. Mori, C. Chen, and S.-W. Cheong, Nature (London) 392, 473 (1998).

${ }^{24}$ P. G. Radaelli, D. E. Cox, L. Capogna, S. W. Cheong, and M. Marezio, Phys. Rev. B 59, 14440 (1999).

${ }^{25}$ D. I. Khomskii and K. I. Kugel, Phys. Rev. B 67, 134401 (2003).

${ }^{26}$ T. Hotta, A. Feiguin, and E. Dagotto, Phys. Rev. Lett. 86, 4922 (2001).

${ }^{27}$ L. Brey, Phys. Rev. Lett. 92, 127202 (2004).

${ }^{28}$ T. Maitra and A. Taraphder, Europhys. Lett. 65, 262 (2004).

${ }^{29}$ Z. P. Luo, D. J. Miller, and J. F. Mitchell, Phys. Rev. B 71, 014418 (2005). 